\title{
A General Relativistic Magnetohydrodynamic Model of High Frequency Quasi-periodic Oscillations in Black Hole Low-Mass X-ray Binaries
}

\author{
Chang-Sheng Shi ${ }^{1,2,3}$ and Xiang-Dong $\mathrm{Li}^{1,2}$
}

\begin{abstract}
We suggest a possible explanation for the high frequency quasi-periodic oscillations (QPOs) in black hole low mass X-ray binaries. By solving the perturbation general relativistic magnetohydrodynamic equations, we find two stable modes of the Alfven wave in the the accretion disks with toroidal magnetic fields. We suggest that these two modes may lead to the double high frequency QPOs if they are produced in the transition region between the inner advection dominated accretion flow and the outer thin disk. This model naturally accounts for the $3: 2$ relation for the upper and lower frequencies of the QPOs, and the relation between the black hole mass and QPO frequency.

Subject headings: magnetohydrodynamics - QPOs - accretion disc - stars: black hole
\end{abstract}

\section{Introduction}

Low-mass X-ray binaries (LMXBs) are binary systems consisting of a neutron star (NS) or black hole $(\mathrm{BH})$ accreting from a low-mass $\left(\lesssim 1 M_{\odot}\right)$ companion star. X-ray emission of LMXBs often shows fast X-ray variability in the form of high frequency quasi-periodic oscillations (HFQPOs), which frequently appear in pairs in certain state simultaneously (van der Klis 2006). Abramowicz \& Kluźniak (2001) pointed out that the frequency ratio of the twinpeak HFQPOs in the BH source GRO J1655-40 equals 3/2, and that this commensurability of frequencies may be a signature of a non-linear resonance. Later, Abramowicz et al. (2003)

\footnotetext{
${ }^{1}$ Department of Astronomy, Nanjing University, Nanjing 210093, China; E-mail: scs1217@gmail.com, lixd@nju.edu.cn

${ }^{2}$ Key Laboratory of Modern Astronomy and Astrophysics (Nanjing University), Ministry of Education, Nanjing 210093, China

${ }^{3}$ College of Material Science and Chemical Engineering, Hainan University, Hainan 570228, China
} 
found a signature of the same commensurable ratio in the twin-peak HFQPOs observed in an NS-LMXB, Sco X-1. Based on this observational evidence, Kluźniak and Abramowicz argued in several papers (Kluźniak \& Abramowicz 2001; Kluźniak, Abramowicz, \& Lee 2004; Kluźniak et al. 2004) that the twin-peak HFQPOs in both BH and NS sources are due to the same physical mechanism - a non-linear parametric resonance in accretion disk global oscillations.

However, while for the $\mathrm{BH}$ sources the commensurable ratio $3 / 2$ was quickly confirmed and generally accepted (e.g. Remillard \& McClintock 2006), the presence of the same commensurability in the NS sources is denied by several experts (e.g. Boutelier et al., 2009). There is no consensus whether the nature of the twin-peak HFQPOs in the two types of LMXBs is the same. We have proposed a mechanism for the twin kilohertz QPOs in NSLMXBs using the magnetohydrodynamic (MHD) Alfv́en wave oscillations, and the results seem to fit the observation well (Li \& Zhang 2005; Shi \& Li 2009). In this paper we focus on an MHD explanation of the HFQPOs in BH-LMXBs.

Barret et al. (2005) measured the quality factor Q for the HFQPOs measured in the NS-LMXB $4 \mathrm{U}$ 1608-52, and found that $\mathrm{Q} \sim 200$. They argued that such high coherency is impossible to achieve from kinematic effects in orbital motion of hot spots, clumps or other similar features located at the accretion disk surface, because these features are too quickly sheared out by the differential rotation. Although orbital motion cannot explain the the HFQPOs in LMXBs, the frequencies of several fluid oscillatory modes are expressed by the three characteristic orbital frequencies: the "Keplerian" frequency, the "radial" epicyclic frequency, and the "vertical" epicyclic frequency. In the Kerr metric, these three orbital frequencies and the Lense-Thirring "frame-dragging" frequency have been listed (e.g. Perez et al. 1997). Several HFQPOs models use their ratios (in various combinations) to explain the observed 3/2 commensurability. Cui, Zhang, \& Chen (1998) suggested the Lense-Thirring nodal precession frequency near the inner stable circular orbit (ISCO) radius as the lower HFQPO frequency, such as the $300 \mathrm{~Hz}$ QPOs in GRO J1655-40. The relativistic precession model of Stella et al. (1999) applies to both BH and NS sources; the pariastron precession frequency and the Keplerian frequency were taken as the lower and upper frequencies of the twin HFQPOs, respectively, whereas the QPOs at much lower frequencies were interpreted in terms of the Lense-Thirring nodal precession ferquency. Wang et al. (2003, 2005) suggested that a non-axisymmetric magnetic coupling of a rotating $\mathrm{BH}$ with its surrounding accretion disk coexists with the Blandford-Znajek process. The two frequencies were supposed as the Keplerian frequencies of two hotspots, one near the inner edge of the disk and the other somewhere outside, respectively.

Wagoner et al. (2001) considered the modes of the diskoseismic wave, such as g-modes, 
p-modes and c-modes as the explanation of the HFQPOs. They estimated the masses and angular momenta of some BHs with the measured frequencies of the HFQPOs when the gmodes or c-modes were selected. Rezzolla et al. (2003) discussed the inertial-acoustic modes in a small-size torus very close to the horizon of the $\mathrm{BH}$ while the centrifugal and pressure gradients were selected as the only restoring forces. In this model the black hole spin had to be very close to the maximal value to produce the $3: 2$ ratio. Tassive \& Bertschinger (2008) investigated the kinematic density waves in the accretion disks when nothing but the gravity was considered as the restoring force, and several discrete radii were adopted. Several modes in pairs close the ratio $(3: 2)$ could be got, but the correct frequencies couldn't be reproduced. Similar to the parametric resonance models, it is difficult to explain why other modes such as the fundamental frequencies weren't observed except the two modes in pairs.

In modeling HFQPOs in BH-LMXBs two points need to be mentioned. First, in most models the $3: 2$ ratio was often overemphasized and substituted into those models directly. In fact, the $3: 2$ ratio of the twin HFQPOs in BH-LMXBs isn't rigorous but approximate. Second, the HFQPO frequencies were often considered invariable so that these frequencies (168, $113 \mathrm{~Hz}$ and 67, 41Hz in GRS 1915+105, Remillard (2004); 450, $300 \mathrm{~Hz}$ in GRO J165540, Strohmayer (2001a); 276, $184 \mathrm{~Hz}$ in XTE J1550-564, Miller et al. (2001); 240, $165 \mathrm{~Hz}$ in H1743-322, Homan et al. (2005)) were used to estimate the parameters of the BHs such as the masses and the spins. In fact, the frequencies are stable, i.e. there are small varitions in them, rather than invariable (Miller et al. 2001; Remillard et al. 1999; Morgan et al. 1997; Strohmayer 2001b).

This paper is organized as follows. In Section 2 we suggest the basic model and get two stable modes of the Alfv́en wave by solving the general relativistic magnetohydrodynamic (GRMHD) equations of the perturbed plasma in BH accretion disks. In Section 3 we compare the results with the observations and discuss their possible implications.

\section{The GRMHD model of the HFQPOs in BH-LMXBs}

We consider that the HFQPOs of BH-LMXBs result from GRMHD waves caused by perturbations in the disk. According to $\S 2.1$ we can find that only one type of GRMHD wave, the steady Alfv́en wave, can spread along the magnetic field lines to the energy release region. The simulation by Koide (2002) also shows that a torsional Alfv́en wave can be generated by the rotational dragging of space. We assume that the puny disturbance doesn't change the metric of the space-time. 


\subsection{The modes of the GRMHD wave}

The oscillation modes of plasma in accretion disks with or without magnetic field, such as g-modes, p-modes, and c-modes, have ever been investigated (Wagoner et al. 2001; Fu \& Lai 2009; Lai 2009). Here we discuss the GRMHD wave modes in ideal adiabatic magnetofluid. In the fiducial observer (FIDO) frame, which is a locally inertial frame, the line element can be written as $(\mathrm{d} s)^{2}=-(c \mathrm{~d} t)^{2}+\sum_{i=1}^{3}\left(\mathrm{~d} x^{i}\right)^{2}$, where $c$ is the speed of light in vacuum, the Roman indices $(i)$ run from 1 to 3 , and $\left(c t, x^{1}, x^{2}, x^{3}\right)$ are the coordinates of the FIDO frame. The metric of the Kerr space-time in the Boyer-Lindquist coordinates $\left(c t^{\prime}, r, \theta, \varphi\right)$ is introduced and the line element for the observer at infinity (i.e. in the laboratory frame) can be written as,

$$
(\mathrm{d} s)^{2}=-h_{0}^{2}\left(c \mathrm{~d} t^{\prime}\right)^{2}+\sum_{i=1}^{3}\left[h_{i}^{2}\left(\mathrm{~d} x^{i \prime}\right)^{2}-2 h_{i}^{2} \omega_{i} \mathrm{~d} t^{\prime} \mathrm{d} x^{i \prime}\right]
$$

where

$$
h_{0}^{2}=1-\frac{2 r r_{g}}{\Sigma}, h_{1}^{2}=\frac{\Sigma}{\Delta}, h_{2}^{2}=\Sigma, h_{3}^{2}=\frac{A}{\Sigma} \sin ^{2} \theta, \omega_{1}=\omega_{2}=0, \omega_{3}=\frac{2 c a r r_{g}^{2}}{A} .
$$

Here $\left(c t^{\prime}, x^{1 \prime}, x^{2 \prime}, x^{3 \prime}\right)$ are the coordinates of the laboratory frame, $h_{0}, h_{i}$ and $\omega_{i}$ are the metrics of the Kerr space-time, $r_{g}=G M / c^{2}, a=J c / G M^{2}$ ( $M$ and $J$ are the mass and the angular momentum of the $\mathrm{BH}$ respectively), $G$ is gravitational constant, $\Sigma=r^{2}+a^{2} r_{g}^{2} \cos ^{2} \theta$, $\Delta=r^{2}-2 r r_{g}+a^{2} r_{g}^{2}$, and $A=\left(r^{2}+a^{2} r_{g}^{2}\right)^{2}-a^{2} r_{g}^{2} \sin ^{2} \theta \Delta$. Since our discussion is limited to the accretion disk we take $\theta=\pi / 2$ and get $\Sigma=r^{2}$ and $A=\left(r^{2}+a^{2} r_{g}^{2}\right)^{2}-a^{2} r_{g}^{2} \Delta$, where $r$ is the distance of the plasma from the $\mathrm{BH}$. Besides that, the lapse function and the shift velocity can be expressed as,

$$
\alpha=\sqrt{h_{0}^{2}+\sum_{i=1}^{3}\left(\frac{h_{i} \omega_{i}}{c}\right)^{2}}=\sqrt{\frac{r^{4}-2 r^{3} r_{g}+a^{2} r^{2} r_{g}^{2}}{r^{4}+a^{2} r^{2} r_{g}^{2}+2 a^{2} r r_{g}^{3}}}
$$

and

$$
\beta^{i}=\frac{h_{i} \omega_{i}}{c \alpha} \text { or } \boldsymbol{\beta}=\left(\beta_{1}, \beta_{2}, \beta_{3}\right)=\left(0,0, \frac{2 a r_{g}^{2}}{r \sqrt{r^{2}-2 r r_{g}+a^{2} r_{g}^{2}}}\right),
$$

where $\boldsymbol{\beta}$ is a vector parallel to the toroidal velocity of the plasma.

We begin with the form of $3+1$ split of the GRMHD equations as in Koide (2003). Previous investigations (Ruzmaikin et al. 1979; Tout et al. 1992; Ruediger et al. 1995; Hawley et al. 2000; Moss et al. 2004; Hirose et al. 2004) have shown that in the accretion disk around a $\mathrm{BH}$ the toroidal component of the magnetic field may be much stronger than 
the poloidal component, i.e., $B_{r} \ll B_{\varphi}$ and $B_{\theta} \ll B_{\varphi}$. In our analysis we also assume $v_{r} \ll v_{\varphi}$ and $v_{\theta} \ll v_{\varphi}$. The equations in the FIDO frame are then written as follows:

$$
\begin{gathered}
\frac{\partial(\gamma \rho)}{\partial t}=-\nabla \cdot[\alpha \gamma \rho(\mathbf{v}+c \boldsymbol{\beta})], \\
\frac{\partial \mathbf{P}}{\partial t}=-\nabla \cdot[\alpha(\widetilde{\mathbf{T}}+c \boldsymbol{\beta} \mathbf{P})]-\left(\varepsilon+\gamma \rho c^{2}\right) \nabla \alpha+\alpha \mathbf{f}_{\text {curv }}-\mathbf{P} \cdot \widetilde{\sigma} \\
\frac{\partial \varepsilon}{\partial t}=-\nabla \cdot\left[\alpha\left(c^{2} \mathbf{P}-\gamma \rho c^{2} \mathbf{v}+\varepsilon c \boldsymbol{\beta}\right)\right]-(\nabla \alpha) \cdot c^{2} \mathbf{P}-\widetilde{\mathbf{T}}: \widetilde{\sigma} \\
\frac{\partial \mathbf{B}}{\partial t}=-\nabla \times[\alpha(\mathbf{E}-c \boldsymbol{\beta} \times \mathbf{B})], \\
\nabla \cdot \mathbf{B}=0, \\
\mathbf{E}+\mathbf{v} \times \mathbf{B}=0, \\
p \rho^{-\Gamma}=\text { constant, }
\end{gathered}
$$

where $\mathbf{v}$ is the velocity of the plasma, $\rho$ the plasma density, $p$ the barometric pressure, $\Gamma$ the adiabatic index, $\gamma$ the Lorentz factor, $\mathbf{E}=\mathbf{E}^{\prime} / \sqrt{\mu_{\mathbf{0}}}, \mathbf{B}=\mathbf{B}^{\prime} / \sqrt{\mu_{\mathbf{0}}}$ (here $\mathbf{E}^{\prime}$ is the electric field, $\mathbf{B}^{\prime}$ the magnetic field, and $\mu_{0}$ the magnetic permeability in the vacuum), respectively. The bold characters denote vectors, and the superscript $\sim$ corresponds to tensors. The energy-momentum tensor is,

$$
\widetilde{\mathbf{T}}=\left(p+\frac{B^{2}}{2}+\frac{E^{2}}{2 c^{2}}\right) \widetilde{\mathbf{I}}+\frac{\psi}{c^{2}} \gamma^{2} \mathbf{V} \mathbf{V}-\mathbf{B B}-\frac{1}{c^{2}} \mathbf{E E},
$$

where $\psi=\rho c^{2}+\frac{\Gamma p}{\Gamma-1}$ is the relativistic enthalpy density. The equivalent momentum density and energy density are,

$$
\mathbf{P}=\frac{\psi}{c^{2}} \gamma^{2} \mathbf{V}+\frac{1}{c^{2}} \mathbf{E} \times \mathbf{B}
$$

and

$$
\varepsilon=\psi \gamma^{2}-p-\gamma \rho c^{2}+\frac{B^{2}}{2}+\frac{E^{2}}{2 c^{2}}
$$

respectively. Equations (5), (6), (7), (8), and (9) correspond to the continuity equation, the momentum conservation equation, the energy conservation equation, the law of electromagnetic induction, and the equation of no divergence, respectively. Equations (10) and (11) are the equations of state for the infinite electrical conductivity and the adiabatic condition. The other physical quantities are,

$$
\mathbf{f}_{\text {curv }} \equiv \sum_{j}\left(G_{i j} T^{i j}-G_{j i} T^{j j}\right), \sigma_{i j} \equiv\left(h_{i} / h_{j}\right)\left(\partial \omega_{i} / \partial x^{j}\right), G_{i j} \equiv-\left(1 / h_{i} h_{j}\right) \times\left(\partial h_{i} / \partial x^{j}\right) .
$$


We can simplify the above two tensors in the accretion disk in the Kerr space-time as,

$$
\sigma_{i j}=\left\{\begin{array}{lll}
0 & 0 & 0 \\
0 & 0 & 0 \\
-\frac{2 a c r_{g}^{2}\left(3 r^{2}+a^{2} r_{g}^{2}\right) \sqrt{r^{2}+a^{2} r_{g}^{2}+\frac{2 a^{2} r_{g}^{3}}{r}}}{\sqrt{\frac{r^{2}}{r^{2}+a^{2} r_{g}^{2}-2 r r_{g}}}\left(r^{3}+r a^{2} r_{g}^{2}+2 a^{2} r_{g}^{3}\right)^{2}} & 0 & 0
\end{array}\right\}=\left(\begin{array}{l}
0 \\
0 \\
1
\end{array}\right)\left(\begin{array}{llll}
\frac{h_{3}}{h_{1}} \frac{\partial \omega_{3}}{\partial x^{1}} & 0 & 0
\end{array}\right),
$$

and

$$
G_{i j}=-\left\{\begin{array}{lll}
\frac{r_{g}\left(a^{2} r_{g}-r\right)}{r^{2} \sqrt{r^{2}-2 r r_{g}+a^{2} r_{g}^{2}}} & 0 & 0 \\
\frac{\sqrt{r^{2}-2 r r_{g}+a^{2} r_{g}^{2}}}{r^{2}} & 0 & 0 \\
\frac{\left(r^{3}-a^{2} r_{g}^{3}\right) \sqrt{r^{2}-2 r r_{g}+a^{2} r_{g}^{2}}}{\left(r^{3}+r a^{2} r_{g}^{2}+2 a^{2} r_{g}^{3}\right) r^{2}} & 0 & 0
\end{array}\right\},
$$

where we define the vector $\mathbf{N} \equiv\left[\left(h_{3} / h_{1}\right)\left(\partial \omega_{3} / \partial x^{1}\right), 0,0\right]$. The magnetized accretion torus with a toroidal magnetic field around a Kerr black hole can exist stably (Komissarov 2006), and the GRMHD equations in the steady state can be expressed as,

$$
\begin{gathered}
\frac{\partial\left(\gamma \rho_{0}\right)}{\partial t}=-\nabla \cdot\left[\alpha \gamma \rho_{0}\left(\mathbf{v}_{0}+c \boldsymbol{\beta}\right)\right] \\
\frac{\partial \mathbf{P}_{0}}{\partial t}=-\nabla \cdot\left[\alpha\left(\widetilde{\mathbf{T}}_{0}+c \boldsymbol{\beta} \mathbf{P}_{0}\right)\right]-\left(\varepsilon_{0}+\gamma \rho_{0} c^{2}\right) \nabla \alpha+\alpha \mathbf{f}_{\mathrm{curv}, 0}-\mathbf{P}_{0} \cdot \widetilde{\sigma} \\
\frac{\partial \varepsilon_{0}}{\partial t}=-\nabla \cdot\left[\alpha\left(c^{2} \mathbf{P}_{0}-\gamma \rho_{0} c^{2} \mathbf{v}_{0}+\varepsilon_{0} c \boldsymbol{\beta}\right)\right]-(\nabla \alpha) \cdot c^{2} \mathbf{P}_{0}-\widetilde{\mathbf{T}}_{0}: \widetilde{\sigma} \\
\frac{\partial \mathbf{B}_{0}}{\partial t}=-\nabla \times\left[\alpha\left(\mathbf{E}_{0}-c \boldsymbol{\beta} \times \mathbf{B}_{0}\right)\right] \\
\nabla \cdot \mathbf{B}_{0}=0 \\
\mathbf{E}_{0}+\mathbf{v}_{0} \times \mathbf{B}_{0}=0 \\
p_{0} \rho_{0}^{-\Gamma}=\text { constant }
\end{gathered}
$$

where the subscript 0 denotes the variables in steady state. Next we consider the GRMHD equations after the plasma is perturbed slightly,

$$
\begin{gathered}
\frac{\partial(\gamma \hat{\rho})}{\partial t}=-\nabla \cdot[\alpha \gamma \hat{\rho}(\hat{\mathbf{v}}+c \boldsymbol{\beta})], \\
\frac{\partial \hat{\mathbf{P}}}{\partial t}=-\nabla \cdot[\alpha(\hat{\widetilde{\mathbf{T}}}+c \boldsymbol{\beta} \hat{\mathbf{P}})]-\left(\hat{\varepsilon}+\gamma \hat{\rho} c^{2}\right) \nabla \alpha+\alpha \hat{\mathbf{f}}_{\mathrm{curv}}-\hat{\mathbf{P}} \cdot \widetilde{\sigma} \\
\frac{\partial \hat{\varepsilon}}{\partial t}=-\nabla \cdot\left[\alpha\left(c^{2} \hat{\mathbf{P}}-\gamma \hat{\rho} c^{2} \hat{\mathbf{v}}+\hat{\varepsilon} c \boldsymbol{\beta}\right)\right]-(\nabla \alpha) \cdot c^{2} \hat{\mathbf{P}}-\hat{\widetilde{\mathbf{T}}}: \widetilde{\sigma}
\end{gathered}
$$




$$
\begin{gathered}
\frac{\partial \hat{\mathbf{B}}}{\partial t}=-\nabla \times[\alpha(\hat{\mathbf{E}}-c \boldsymbol{\beta} \times \hat{\mathbf{B}})], \\
\nabla \cdot \hat{\mathbf{B}}=0, \\
\hat{\mathbf{E}}+\hat{\mathbf{v}} \times \hat{\mathbf{B}}=0, \\
\hat{p} \hat{\rho}^{-\Gamma}=p_{0} \rho_{0}^{-\Gamma},
\end{gathered}
$$

where $\hat{\boldsymbol{v}}=\boldsymbol{v}_{0}+\boldsymbol{v}_{s}, \hat{\rho}=\rho_{0}+\rho_{s}, \hat{p}=p_{0}+p_{s}, \hat{\varepsilon}=\varepsilon_{0}+\varepsilon_{s}, \hat{\boldsymbol{E}}=\boldsymbol{E}_{0}+\boldsymbol{E}_{s}, \hat{\boldsymbol{B}}=\boldsymbol{B}_{\mathbf{0}}+\boldsymbol{B}_{s}$, $\hat{\boldsymbol{P}}=\boldsymbol{P}_{0}+\boldsymbol{P}_{s}, \hat{\boldsymbol{f}}_{\text {curv }}=\boldsymbol{f}_{\text {curv }, 0}+\boldsymbol{f}_{\text {curv }, s}$, and $\hat{\tilde{\boldsymbol{T}}}=\widetilde{\boldsymbol{T}}_{0}+\widetilde{\boldsymbol{T}}_{s}$, with the subscript $s$ denoting the perturbed quantities $\left(v_{s} \ll v_{0}, E_{s} \ll E_{0}, B_{s} \ll B_{0}, \rho_{s} \ll \rho_{0}\right.$, and $\left.p_{s} \ll p_{0}\right)$ and with the superscript ^denoting the variables after the disturbance. By combining Eqs. (18)-(31) we get the equations about the perturbed quantities in the first-order approximation,

$$
\begin{gathered}
\frac{\partial\left(\gamma \rho_{s}\right)}{\partial t}=-\nabla \cdot\left[\alpha \gamma\left(\rho_{0} \mathbf{v}_{s}+\rho_{s} \mathbf{v}+\rho_{s} c \beta\right)\right] \\
\frac{\partial \mathbf{P}_{s}}{\partial t}=-\nabla \cdot\left[\alpha\left(\widetilde{\mathbf{T}}_{s}+c \beta \mathbf{P}_{s}\right)\right]-\left(\varepsilon_{s}+\gamma \rho_{s} c^{2}\right) \nabla \alpha+\alpha f_{\mathrm{curv}, s}-\mathbf{P}_{s} \cdot \sigma \\
\frac{\partial \varepsilon_{s}}{\partial t}=-\nabla \cdot\left[\alpha\left(c^{2} \mathbf{P}_{s}-\gamma c^{2} \rho_{0} \mathbf{v}_{s}-\gamma c^{2} \rho_{s} \mathbf{v}_{0}+\varepsilon_{s} c \beta\right)\right]-(\nabla \alpha) \cdot c^{2} \mathbf{P}_{s}-\mathbf{T}_{s}: \sigma, \\
\frac{\partial \mathbf{B}_{s}}{\partial t}=-\nabla \times\left[\alpha\left(\mathbf{E}_{s}-c \beta \times \mathbf{B}_{s}\right)\right], \\
\nabla \cdot \mathbf{B}_{s}=0, \\
\mathbf{E}_{s}+\mathbf{v}_{0} \times \mathbf{B}_{s}+\mathbf{v}_{s} \times \mathbf{B}_{0}=0, \\
p_{s}=\frac{\Gamma p_{0}}{\rho_{0}} \rho_{s} .
\end{gathered}
$$

With Eqs. (12)-(14) we can get the perturbed energy-momentum tensor $\left(\widetilde{\boldsymbol{T}}_{s}\right)$, equivalent momentum density $\left(\boldsymbol{P}_{s}\right)$ and equivalent energy density $\left(\boldsymbol{\varepsilon}_{s}\right)$ in the same way, by defining $\psi_{0}=\rho_{0} c^{2}+\frac{\Gamma p_{0}}{\Gamma-1}$ and $\psi_{s}=\rho_{s} c^{2}+\frac{\Gamma p_{s}}{\Gamma-1}$,

$$
\begin{array}{r}
\widetilde{\mathbf{T}}_{s}=\left(p_{s}+\mathbf{B}_{0} \cdot \mathbf{B}_{s}+\frac{1}{c^{2}} \mathbf{E}_{0} \cdot \mathbf{E}_{s}\right) \widetilde{\mathbf{I}}+\frac{\psi_{\mathrm{s}}}{c^{2}} \gamma^{2} \mathbf{V}_{0} \mathbf{V}_{0}+\frac{\psi_{0}}{c^{2}} \gamma^{2}\left(\mathbf{V}_{0} \mathbf{V}_{\mathrm{s}}+\mathbf{V}_{\mathrm{s}} \mathbf{V}_{0}\right) \\
-\left(\mathbf{B}_{0} \mathbf{B}_{s}+\mathbf{B}_{s} \mathbf{B}_{0}\right)-\frac{1}{c^{2}}\left(\mathbf{E}_{0} \mathbf{E}_{s}+\mathbf{E}_{s} \mathbf{E}_{0}\right), \\
\mathbf{P}_{\mathrm{s}}=\frac{\gamma^{2}}{c^{2}}\left(\psi_{0} \mathbf{v}_{s}+\psi_{s} \mathbf{v}_{0}\right)+\frac{1}{c^{2}} \mathbf{E}_{s} \times \mathbf{B}_{0}+\frac{1}{c^{2}} \mathbf{E}_{0} \times \mathbf{B}_{s}, \\
\varepsilon=\psi_{s} \gamma^{2}-p_{s}-\gamma \rho_{s} c^{2}+\mathbf{B}_{0} \cdot \mathbf{B}_{s}+\frac{\mathbf{E}_{0} \cdot \mathbf{E}_{s}}{c^{2}}
\end{array}
$$


After carrying out Fourier transformation $\left(e^{i \mathbf{k} \cdot \mathbf{r}-i \omega t}\right)$ for Eqs. (32)-(36) and substituting Eqs. (37) and (38) into them, we get the following equations when $\mathbf{v}_{0}\left\|\mathbf{B}_{0}\right\| \boldsymbol{\beta}$ is considered,

$$
\begin{gathered}
{\left[\omega-\alpha\left(\mathbf{k} \cdot \mathbf{v}_{0}\right)-\alpha c(\mathbf{k} \cdot \boldsymbol{\beta})\right] \rho_{s}=\alpha \rho_{0}\left(\mathbf{k} \cdot \mathbf{v}_{s}\right),} \\
\omega \mathbf{P}_{s}-\alpha c(\mathbf{k} \cdot \boldsymbol{\beta}) \mathbf{P}_{s}=\alpha \mathbf{k} \cdot \widetilde{\mathbf{T}}_{s}+\alpha\left(\varepsilon_{s}+\gamma \rho_{s} c^{2}\right) \mathbf{k}+i \alpha \mathbf{f}_{\mathrm{curv}, s}-i \mathbf{P}_{s} \cdot \widetilde{\sigma} \\
\omega \varepsilon_{s}-\alpha c(\mathbf{k} \cdot \boldsymbol{\beta}) \varepsilon_{s}=2 \alpha c^{2}\left(\mathbf{k} \cdot \mathbf{P}_{s}\right)-\alpha \gamma c^{2} \rho_{0}\left(\mathbf{k} \cdot \mathbf{v}_{s}\right)-\alpha \gamma c^{2}\left(\mathbf{k} \cdot \mathbf{v}_{0}\right) \rho_{s}-i \widetilde{\mathbf{T}}_{s}: \widetilde{\sigma} \\
{\left[\omega-\alpha\left(\mathbf{k} \cdot \mathbf{v}_{0}\right)-\alpha c(\mathbf{k} \cdot \boldsymbol{\beta})\right] \mathbf{B}_{s}=\alpha\left(\mathbf{k} \cdot \mathbf{v}_{s}\right) \mathbf{B}_{0}-\alpha\left(\mathbf{k} \cdot \mathbf{B}_{0}\right) \mathbf{v}_{s},} \\
\mathbf{k} \cdot \mathbf{B}_{s}=0,
\end{gathered}
$$

where $\mathbf{k}$ is the wave vector and $\omega$ is the the oscillation frequency. When Eqs. (37) and (38) are substituted into Eqs. (39)-(41) and $\mathbf{v}_{0}\left\|\mathbf{B}_{0}\right\| \boldsymbol{\beta}$ is considered, Eqs. (39)-(41) can be converted to be,

$$
\begin{gathered}
\widetilde{\mathbf{T}}_{s}=\left(\frac{\Gamma p_{0}}{\rho_{0}} \rho_{s}+\mathbf{B}_{0} \cdot \mathbf{B}_{s}\right) \widetilde{\mathbf{I}}+\frac{\psi_{\mathrm{s}}}{c^{2}} \gamma^{2} \mathbf{V}_{0} \mathbf{V}_{0}+\frac{\psi_{0}}{c^{2}} \gamma^{2}\left(\mathbf{V}_{0} \mathbf{V}_{s}+\mathbf{V}_{s} \mathbf{V}_{0}\right)-\left(\mathbf{B}_{0} \mathbf{B}_{s}+\mathbf{B}_{s} \mathbf{B}_{0}\right), \\
\mathbf{P}_{\mathrm{s}}=\frac{\gamma^{2}}{c^{2}}\left(\psi_{0} \mathbf{v}_{s}+\psi_{s} \mathbf{v}_{0}\right)-\frac{1}{c^{2}}\left(\mathbf{v}_{0} \cdot \mathbf{B}_{0}\right) \mathbf{B}_{s}+\frac{1}{c^{2}}\left(\mathbf{B}_{s} \cdot \mathbf{B}_{0}\right) \mathbf{v}_{0}-\frac{1}{c^{2}}\left(\mathbf{v}_{s} \cdot \mathbf{B}_{0}\right) \mathbf{B}_{0}+\frac{B_{0}^{2}}{c^{2}} \mathbf{v}_{s}, \\
\varepsilon=\psi_{s} \gamma^{2}-\frac{\Gamma p_{0}}{\rho_{0}} \rho_{s}-\gamma \rho_{s} c^{2}+\mathbf{B}_{0} \cdot \mathbf{B}_{s} .
\end{gathered}
$$

If $\omega=\alpha \mathbf{k} \cdot \mathbf{v}_{0}+\alpha c \mathbf{k} \cdot \boldsymbol{\beta}$, we get a unphysical solution $(\omega=0)$ from Eqs. (42)-(49), because the conditions $\mathbf{k} \cdot \mathbf{v}_{s}=0$ and $\mathbf{k} \cdot \mathbf{v}_{0}=0$ are derived from Eqs. (42)-(44). Now we consider $\omega \neq \alpha \mathbf{k} \cdot \mathbf{v}_{0}+\alpha c \mathbf{k} \cdot \boldsymbol{\beta}$ and discuss all the three types of MHD waves: the Alfíen wave, the ion-acoustic wave, and the magnetosonic wave.

\subsubsection{The Alfúen wave}

The transportation direction of Alfv́en waves is along the magnetic field line, so $\mathbf{k}\left\|\mathbf{v}_{0}\right\| \mathbf{B}_{0} \| \boldsymbol{\beta}$, where $\|$ denotes parallel and $\overrightarrow{\|}$ the same direction. Since the Alfven wave is a transverse wave, i.e. $\mathbf{k} \perp \mathbf{B}_{s}, \mathbf{k} \perp \mathbf{v}_{s}$ or the $\varphi$ component of the perturbed velocity, $v_{s, \varphi}=0$.

From Eqs. (15)-(17), (42), (47), and (48) and the above results we obtain

$$
\begin{gathered}
\mathbf{f}_{\text {curv }, s}=(0,0,0) \\
\mathbf{P}_{s} \cdot \widetilde{\sigma}=\frac{\gamma^{2}}{c^{2}}\left(\psi_{0} v_{s, \varphi}+\psi_{s} v_{0}\right) \mathbf{N}=0
\end{gathered}
$$

and

$$
\widetilde{\mathbf{T}}: \widetilde{\sigma}=\sigma_{31}\left[\frac{\gamma^{2}}{c^{2}} \psi_{0} v_{0}+\frac{\alpha k B_{0}^{2}}{\omega-\alpha\left(\mathbf{k} \cdot \mathbf{v}_{0}\right)-\alpha c(\mathbf{k} \cdot \boldsymbol{\beta})}\right] v_{s, \mathrm{r}},
$$


where $v_{s, \mathrm{r}}$ denotes the $r$ component of the perturbed velocity. Substituting the above equations and Eqs. (42), (45)-(49) into Eqs. (43) and (44) we can get $v_{s, \mathrm{r}}=0$ and the dispersion equation,

$$
[\omega-\alpha c(\mathbf{k} \cdot \boldsymbol{\beta})]^{2}\left(\gamma^{2} \psi_{0}+B_{0}^{2}\right)-\left(2 \alpha \gamma^{2} \psi_{0} v_{0} k\right)[\omega-\alpha c(\mathbf{k} \cdot \boldsymbol{\beta})]+\alpha^{2} \gamma^{2} \psi_{0}\left(k^{2} v_{0}^{2}\right)-\alpha^{2} c^{2} k^{2} B_{0}^{2}=0 .
$$

The frequencies of the Alfv́en waves are solved as,

$$
\omega=k \alpha\left[\beta_{3} c+\frac{\gamma^{2} \psi_{0} v_{0} \pm B_{0} \sqrt{B_{0}^{2} c^{2}+\left(c^{2}-v_{0}^{2}\right) \gamma^{2} \psi_{0}}}{B_{0}^{2}+\gamma^{2} \psi_{0}}\right] .
$$

The group velocities of the Alfven waves are in the same form of the phase velocities of the Alfv́en waves and are,

$$
\frac{\omega}{k}=\frac{d \omega}{d k}=\alpha\left[\beta_{3} c+\frac{\gamma^{2} \psi_{0} v_{0} \pm B_{0} \sqrt{B_{0}^{2} c^{2}+\left(c^{2}-v_{0}^{2}\right) \gamma^{2} \psi_{0}}}{B_{0}^{2}+\gamma^{2} \psi_{0}}\right] .
$$

These velocities can be simplified in the special relativity, i.e. $r \rightarrow \infty, \beta_{3} \rightarrow 0 \& \alpha \rightarrow 1$, as

$$
v_{\mathrm{A}}=\frac{\gamma^{2} v_{0} \pm \sqrt{\frac{B_{0}^{2}}{\psi_{0}}+\left(\frac{B_{0}^{2}}{\psi_{0}}\right)^{2}} c}{\frac{B_{0}^{2}}{\psi_{0}}+\gamma^{2}}=\frac{v_{0} \pm \eta \sqrt{\frac{1}{\gamma^{2}}+\eta^{2}} c}{\eta^{2}+1}
$$

where $\gamma^{2} \eta^{2}=B_{0}^{2} / \psi_{0}$. Equation (53) is in the same form as the expression of De Villiers \& Hawley (2003).

\subsubsection{The ion-acoustic wave and the magnetosonic wave}

The ion-acoustic wave is a longitudinal wave without electromagnetic polarization so $\mathbf{k} \| \mathbf{v}_{s}$ and $B_{s}=0$. According to Eq. (45) we have $\mathbf{B}_{0} \| \mathbf{V}_{s}$ owing to $B_{s}=0$. This leads to the conclusion that $\mathbf{k}\left\|\mathbf{v}_{s}\right\| \mathbf{B}_{0}\left\|\mathbf{v}_{0}\right\| \boldsymbol{\beta}$, and that no ion-acoustic wave solution fits Eqs. (42)-(49).

The magnetosonic wave is another type of longitudinal wave with transverse electromagnetic polarization, i.e., $\mathbf{k} \| \mathbf{v}_{s}, \mathbf{k} \perp \mathbf{B}_{s}$. The wave vector $\mathbf{k}$ isn't parallel with $\mathbf{B}_{0}$ under the condition $\mathbf{B}_{0}\left\|\mathbf{v}_{0}\right\| \boldsymbol{\beta}$, otherwise the wave is degenerated into the ion-acoustic wave and does not exist. Now we discuss the magnetosonic wave under two different conditions respectively.

Firstly when $\mathbf{k} \perp \mathbf{v}_{0}$ we get $\mathbf{k}\left\|\mathbf{v}_{s}, \mathbf{k} \perp \mathbf{B}_{s}, \mathbf{B}_{0}\right\| \mathbf{v}_{0} \| \boldsymbol{\beta} \perp \mathbf{k}$, and Eqs. (42)-(49) can be simplified to be,

$$
\left(\alpha \gamma^{2} \psi_{0}+\alpha \Gamma p_{0}-\alpha \gamma^{2} \Gamma p_{0}+\alpha B_{0}^{2}\right)\left(\mathbf{k} \cdot \mathbf{v}_{\mathbf{s}}\right)=i \widetilde{\mathbf{T}}_{s}: \widetilde{\sigma}
$$




$$
\begin{gathered}
\omega^{2}\left(\gamma^{2} \psi_{0}+B_{0}^{2}\right) \mathbf{v}_{s}+\gamma^{2} \alpha \Gamma p_{0}\left(\mathbf{k} \cdot \mathbf{v}_{s}\right) \omega \mathbf{v}_{0}-\alpha^{2} c^{2} k^{2}\left(2 B_{0}^{2}+\gamma^{2} \rho_{0} c^{2}+\gamma^{2} \frac{\Gamma^{2}}{\Gamma-1} p_{0}\right) \mathbf{v}_{s}=i c^{2} \omega\left(\alpha \mathbf{f}_{\mathrm{curv}, s}-\mathbf{P}_{s} \cdot \widetilde{\sigma}\right) \\
\widetilde{\mathbf{T}}_{s}: \widetilde{\sigma}=\frac{\gamma^{2}}{c^{2}} \frac{h_{3}}{h_{1}} \frac{\partial \omega_{3}}{\partial x^{1}} \psi_{0} v_{0} v_{s, \mathrm{r}} \\
\mathbf{P}_{s} \cdot \widetilde{\sigma}=\frac{\gamma^{2}}{c^{2}} \alpha\left(\rho_{0} c^{2}+\frac{\Gamma^{2}}{\Gamma-1} p_{0}\right) \frac{v_{0}}{\omega}\left(\mathbf{k} \cdot \mathbf{v}_{s}\right) \mathbf{N}
\end{gathered}
$$

where

$$
\mathbf{f}_{\mathrm{curv}, s}=\left(f_{s, \mathrm{r}}, 0, G_{31} \frac{\gamma^{2}}{c^{2}} \psi_{0} v_{0} v_{s, \mathrm{r}}\right)
$$

with

$$
f_{s, \mathrm{r}}=-G_{21} \alpha\left(\mathbf{k} \cdot \mathbf{v}_{s}\right) \frac{\Gamma p_{0}+B_{0}^{2}}{\omega}-G_{31}\left[\alpha \frac{\Gamma p_{0}-B_{0}^{2}}{\omega}+\frac{\gamma^{2}}{c^{2}} \alpha\left(\rho_{0} c^{2}+\frac{\Gamma^{2}}{\Gamma-1} p_{0}\right) \frac{v_{0}^{2}}{\omega}\right]\left(\mathbf{k} \cdot \mathbf{v}_{s}\right) .
$$

From Eqs. (54)-(58) we can obtain an unstable solution (an increasing wave or an attenuation wave) if

$$
\frac{\widetilde{\mathbf{T}}_{s}: \widetilde{\sigma}}{\alpha \gamma^{2} \psi_{0}+\alpha \Gamma p_{0}-\alpha \gamma^{2} \Gamma p_{0}+\alpha B_{0}^{2}}=-\frac{c^{2} f_{s, \varphi}}{\Gamma \gamma^{2} p_{0} v_{0}}=\frac{\mathbf{k} \cdot \mathbf{v}_{s}}{i}
$$

or no solution when $\mathbf{k} \perp \mathbf{v}_{0}$. The possible unstable solution is

$$
\omega=\frac{A+\sqrt{A^{2}+4\left(\gamma^{2} \psi_{0}+B_{0}^{2}\right)\left(2 B_{0}^{2}+\gamma^{2} \rho_{0} c^{2}+\frac{\gamma^{2} \Gamma^{2}}{\Gamma-1} p_{0}\right) \alpha^{2} c^{2} k^{2}}}{2 \gamma^{2} \psi_{0}+2 B_{0}^{2}},
$$

where

$$
A=\frac{c^{2} f_{s, \varphi}}{\Gamma \gamma^{2} p_{0} v_{0} v_{s}} \frac{c^{2} \alpha f_{\mathrm{s}, \mathrm{r}}-c^{2}\left(\mathbf{P}_{\mathrm{s}} \cdot \tilde{\sigma}\right)_{\mathrm{r}}}{\mathbf{k} \cdot \mathbf{v}_{s}}
$$

and $\left(\mathbf{P}_{s} \cdot \tilde{\sigma}\right)_{\mathrm{r}}$ represents the $r$ component of the vector $\mathbf{P}_{s} \cdot \widetilde{\sigma}$.

Secondly when $\mathbf{k}$ is neither parallel with nor vertical to $\mathbf{v}_{0}$ we get the solution from Eq. (42)-(49) as follows,

$$
\omega=\alpha c(\mathbf{k} \cdot \boldsymbol{\beta})-\frac{i \alpha\left(\mathbf{k} \cdot \mathbf{v}_{0}\right)\left(\widetilde{\mathbf{T}}_{s}: \widetilde{\sigma}\right)+2 \alpha^{2} \gamma^{2} \Gamma p_{0}\left(\mathbf{k} \cdot \mathbf{v}_{s}\right)\left(\mathbf{k} \cdot \mathbf{v}_{0}\right)}{\left(\alpha \gamma^{2} \psi_{0}+\alpha \Gamma p_{0}-\alpha \gamma^{2} \Gamma p_{0}+\alpha B_{0}^{2}\right)\left(\mathbf{k} \cdot \mathbf{v}_{s}\right)-\alpha\left(\mathbf{k} \cdot \mathbf{B}_{0}\right)\left(\mathbf{B}_{0} \cdot \mathbf{v}_{s}\right)-i \widetilde{\mathbf{T}}_{s}: \widetilde{\sigma}}
$$

which is also unstable.

We summarize the results in $\S 2.1 .1$ and $\S 2.1 .2$ : (1) there are two stable Alfv́en wave modes, (2) the ion-acoustic wave doesn't exist, and (3) a few unstable modes of magnetoacoustic wave may emerge in the GRMHD in the BH accretion disks. 


\subsection{The relation between the magnetic energy density and the relativistic enthalpy density}

It is widely believed that the toroidal magnetic field in accretion disks is generated by dynamo mechanism (Ruzmaikin et al. 1979; Tout et al. 1992; Ruediger et al. 1995; Hawley et al. 2000; Moss et al. 2004), and that accretion is driven by the magnetic stress (e.g. Matsumoto \& Tajima 1995; Brandenburg et al. 1995; Stone et al. 1996). Accordingly the angular momentum conservation gives (Torkelsson 1998)

$$
\dot{M} r v_{\varphi}=2 \pi r H r \frac{B_{\varphi}^{\prime} B_{\mathrm{r}}^{\prime}}{\mu_{0}},
$$

where $H$ is the thickness of the accretion disk, $B_{\varphi}^{\prime}$ and $B_{r}^{\prime}$ are the $\varphi$ - and $r$-components of the magnetic field $B^{\prime}, \dot{M}\left(=2 \pi H r v_{r} \rho\right)$ is the accretion rate, $v_{\varphi}$ and $v_{r}$ are the $\varphi$ - and $r$-components of the velocity of the accreting plasma, respectively. Suppose $B_{\varphi}^{\prime}=\gamma_{\mathrm{dyn}} B_{r}^{\prime}$ and $v_{r}=l v_{\varphi}$, the above equation can be simplified to be,

$$
B_{\varphi}^{\prime 2}=l v_{\varphi}^{2} \rho \mu_{0} \gamma_{\mathrm{dyn}}
$$

The velocity $v_{\varphi}$ can be approximatively expressed as the velocity of the circular orbit relative to Bardeen observers, which can be got from the equations (8.354-8.359) of Camenzind (2007). The velocity of the plasm in the prograde orbit is,

$$
v_{\varphi}=\left[\left(i^{2}+a_{*}^{2}+\frac{2}{i} a_{*}^{2}\right) \frac{i^{2}+a_{*}^{2}-2 i}{i^{3} \sqrt{i}-2 i^{2} \sqrt{i}+a_{*}^{2} i \sqrt{i}+a_{*} i^{2}+a_{*}^{3}-2 a_{*} i}-\frac{2}{i} a_{*}\right] \frac{c}{\sqrt{i^{2}-2 i+a_{*}^{2}}},
$$

and in the retrograde orbit,

$$
v_{\varphi}=\left[\left(i^{2}+a_{*}^{2}+\frac{2}{i} a_{*}^{2}\right) \frac{4 a^{*} \sqrt{i}-\left(i^{2}+a_{*}^{2}-2 i\right)}{i^{3} \sqrt{i}-2 i^{2} \sqrt{i}+a_{*}^{2} i \sqrt{i}-a_{*} i^{2}-a_{*}^{3}+2 a_{*} i}-\frac{2}{i} a_{*}\right] \frac{c}{\sqrt{i^{2}-2 i+a_{*}^{2}}},
$$

where $a_{*}=|a|, i=r / r_{\mathrm{g}}$. The two velocities return to the Keplerian velocity $c / \sqrt{i}$ when $i \rightarrow \infty$.

Since $\frac{\Gamma}{\Gamma-1} p_{0} \ll \rho_{0} c^{2}$ in the accretion disks, we can express $\frac{B_{\varphi}^{\prime 2}}{\mu_{0}}=\gamma^{2} \eta^{2}\left(\rho_{0} c^{2}+\frac{\Gamma p_{0}}{\Gamma-1}\right)$ as $\frac{B_{\varphi}^{\prime 2}}{\mu_{0}}=B_{\varphi}^{2} \simeq \gamma^{2} \eta^{2} \rho_{0} c^{2}$ and $\gamma^{2} \eta^{2}=\gamma_{\mathrm{dyn}} l v_{\varphi}^{2} / c^{2}=l^{\prime} v_{\varphi}^{2} / c^{2}$ from Eq. (62), where $l^{\prime}=\gamma_{\mathrm{dyn}} l=$ $\frac{B_{\varphi}^{\prime}}{B_{\mathrm{r}}^{\prime}} \frac{v_{\mathrm{r}}}{v_{\varphi}}$. We then get the relation between the magnetic energy density and the relativistic enthalpy density, $\frac{B_{\varphi}^{\prime 2}}{2 \mu_{0}} \simeq \frac{l \gamma_{\mathrm{dyn}}}{2} \frac{v_{\varphi}^{2}}{c^{2}} \psi_{0}$, and express the frequencies of the Alfv́en waves, i.e., Eq. (51), as,

$$
\omega=k \alpha\left[\beta_{3} c+\frac{\gamma^{2} \pm \sqrt{l^{\prime}+l^{\prime 2} \frac{v_{\varphi}^{2}}{c^{2}}}}{\gamma^{2}+l^{\prime} \frac{v_{\varphi}^{2}}{c^{2}}} v_{\varphi}\right] .
$$


When we consider the characteristic wavelength $\lambda \sim r$, i.e. the wave number $k \sim 2 \pi / r$, Eq. (65) is reduced to be

$$
\omega \simeq \frac{2 \pi}{r} c \xi=\frac{2 \pi}{i r_{g}} c \xi
$$

where $\xi=\alpha\left[\beta_{3}+\frac{\gamma^{2} \pm \sqrt{l^{\prime}+l^{\prime 2} v_{\varphi}^{2} / c^{2}}}{\gamma^{2} \pm l^{\prime} v_{\varphi}^{2} / c^{2}} \frac{v_{\varphi}}{c}\right]$

\subsection{Estimate of the parameter $l^{\prime}$}

Begelman and Pringle (2007) have investigated the structure of accretion disks with strong toroidal magnetic fields, and found that the thickness of the disks is higher than standard thin disks, but in line with observations (Robinson et al. 1999; Shafter \& Misselt 2006),

$$
\frac{H}{r}=0.48 \alpha_{*}^{-1 / 17} r_{10}^{9 / 68} \dot{M}_{18}^{3 / 34}\left(\frac{M}{M_{\odot}}\right)^{-15 / 68}
$$

where $\alpha_{*}$ is the viscosity prescription, $\dot{M}_{18}=\dot{M} / 10^{18} \mathrm{gs}^{-1}$, and $r_{10}=r / 10^{10} \mathrm{~cm}$. The corresponding radial velocity is,

$$
v_{\mathrm{r}} \simeq \frac{3}{2} \alpha_{*}\left(\frac{H}{r}\right)^{2} v_{\mathrm{k}}
$$

where $v_{\mathrm{k}}$ is the Keplerian velocity. Here we adopt that $v_{\varphi} \simeq v_{\mathrm{k}}$ to estimate the value of $l^{\prime}$. From Eqs. (67) and (68), the parameter $l$ is,

$$
l=\frac{v_{\mathrm{r}}}{v_{\varphi}} \simeq 0.3456 \alpha_{*}^{15 / 17} r_{10}^{9 / 34} \dot{M}_{18}^{3 / 17}\left(\frac{M}{M_{\odot}}\right)^{-15 / 34} .
$$

Vishniac et al.(1990) have discussed the dynamo action by internal waves in accretion disks and suggested $B_{\mathrm{r}} / B_{\varphi} \sim \alpha_{*}$, so we have

$$
l^{\prime}=l \gamma_{\mathrm{dyn}} \simeq 0.3456 \alpha_{*}^{-2 / 17} r_{10}^{9 / 34} \dot{M}_{18}^{3 / 17}\left(\frac{M}{M_{\odot}}\right)^{-15 / 34} .
$$

The relatively small values of the power indices in Eq. (70) indicate that $l^{\prime}$ is not sensitively dependent on $\alpha_{*}, r, M$ and $\dot{M}$.

HFQPOs in BH-LMXBs were generally observed in the steep power-law (SPL) state, i.e. very high state (VHS), and it is very likely that the QPOs are associated with the region that produces hard X-ray emission. The accretion model for the VHS is a long-debated subject. It might contain an inner ADAF surrounded by an outer thin disk (e.g. Yuan 2001). By analyzing the observational data of GRO J1655-40 and XTE J1550-564, McClintock \& Remillard (2006) showed that the disks with blackbody radiation appear to truncate at a 
radius $\left(\sim 100 r_{\mathrm{g}}\right)$ in the low/hard state, and that the truncated radius decreases when the power-law component becomes stronger and steeper. Theoretical investigations (Abramowicz et al. 1995; Honma 1996; Esin et al. 1997; Liu et al. 1999; Manmoto et al. 2000; RóżaŃska et al. 2000; Narayan et al. 2008) also suggest that the transition radius ranges from $\sim 100 r_{\mathrm{g}}$ to $10000 r_{\mathrm{g}}$. Accordingly the transition radius in VHS is likely to be smaller than in the low/hard state, and for illustration here we adopt its value to be $50 r_{\mathrm{g}}$. The X-ray luminosity in the VHS is often more than $0.2 L_{\text {Edd }}$ where $L_{\text {Edd }}$ is the Eddington luminosity (McClintock $\&$ Remillard 2006), so we adopt a typical accretion rate $10^{18} \mathrm{gs}^{-1}$. We also take $\alpha_{*} \sim 0.1$ (King et al. 2007). From the above values we can estimate the parameter $l^{\prime}$ as,

$$
l^{\prime} \sim 0.048\left(\frac{\alpha_{*}}{0.1}\right)^{-2 / 17}\left(\frac{r}{50 r_{\mathrm{g}}}\right)^{9 / 34}\left(\frac{\dot{M}}{10^{18} \mathrm{gs}^{-1}}\right)^{3 / 17}\left(\frac{M}{7 M_{\odot}}\right)^{-15 / 34} .
$$

Combing Eqs. (3), (4), (66), and (71) we get the two frequencies of the HFQPOs as,

$$
\omega=1.2756 \times 10^{6} \frac{\xi}{i}\left(\frac{M}{M_{\odot}}\right)^{-1}
$$

where

$$
\xi=\sqrt{\frac{i^{4}-2 i^{3}+a^{2} i^{2}}{i^{4}+a^{2} i^{2}+2 a^{2} i}}\left[\frac{2 a}{i \sqrt{i^{2}-2 i+a^{2}}}+\frac{\gamma^{2} \pm \sqrt{0.04765+0.0022705 v_{\varphi}^{2} / c^{2}}}{\gamma^{2}+0.04765 v_{\varphi}^{2} / c^{2}} \frac{v_{\varphi}}{c}\right] .
$$

Our calculations show that, for BH-LMXBs with measured HFQPOs the ratio of the two frequencies is generally around 1.5 (see Table 1 ).

\section{Results and discussion}

Equations (63), (64), and (72) indicate the existence of Alfv́en waves with two frequencies in the accretion disk. The ratio of the upper and lower frequencies is close to $3: 2$, suggesting that these waves may account for the HFQPO pairs. Given the mass and spin of a $\mathrm{BH}$, one can determine the radius where the QPOs are produced. In Table 1 we present the inferred radius by comparing Eq. (72) with either the upper or the lower centroid QPO frequency of several BH-LMXBs. We adopt the averaged masses for GRO J1655-40 $\left(6.0-6.6 M_{\odot}\right.$, McClintock \& Remillard 2006), GRS 1915+105 (10 - 18M $\odot$, Greiner et al. 2001), and XTE J1550-564 (8.4 - 10.8M $M_{\odot}$, McClintock \& Remillard 2006) and averaged dimensionless spins of GRO J1655-40 (0.65 - 0.75, Shafee et al. 2006) and GRS 1915+105 (0.98 - 1.0, McClintock \& Shafee et al. 2006). Because the spin of XTE J1550-564 hasn't been measured, we take it to be $1,0.5$, and 0 . It is interesting to see that in most cases the 
radii are $\sim 70 r_{\mathrm{g}}$, consistent with the transition radii between an ADAF and a thin disk discussed above. The frequencies of the HFQPOs mainly depends on the transition radius $\left(r_{\mathrm{tr}}\right)$ and the ratio of $l^{\prime}=\left(v_{\mathrm{r}} / v_{\varphi}\right) /\left(B_{\mathrm{r}}^{\prime} / B_{\varphi}^{\prime}\right)$. According to the discussion in $\S 2.3$ the parameter $l^{\prime}$ changes little with the $\alpha_{*}, r, M$, and $\dot{M}$, this may explain why the HFQPO frequencies are relatively stable during the VHS.

Considering the similarities in $\mathrm{BH}$ and NS accretion disks, it is interesting to ask why the 1.5 frequency ratio is not evident in the HFQPOs in NS-LMXBs. The reasons may lie in the differences in the configuration of the magnetic fields and the structure of the accretion disks in BH- and NS-LMXBs. It is well known that NSs generally hold dipolar magnetic fields, and the toroidal field component, induced by difference in the angular velocity between the disk and the NS magnetosphere, can never be stronger than the poloidal component, otherwise the field configuration becomes unstable leading to inflation of the field lines (e.g. Aly 1985). On the other hand, both theories and simulations (Ruzmaikin et al., 1979; Tout et al., 1992; Ruediger et al., 1995; Hawley et al., 2000; Moss et al, 2004; Hirose et al., 2004)

show that in $\mathrm{BH}$ accretion disks the toroidal magnetic field can be much stronger than the poloidal one. Additionally, recent observations suggest that the inner disk around an NS may not be an ADAF even in low state, as the thermal emission from the surface of the NS would tend to cool an ADAF, making such a flow more difficult than in BH systems (Cackett et al., 2009).

Finally, a correlation between the lower HFQPO frequencies and the BH masses has been suggested, i.e., $\nu_{1} \propto M^{-1}$ (McClintock \& Remillard 2006). This correlation can be naturally reproduced in our model. In Fig. 1 we plot the predicted relation between $\nu_{1}$ and $m$ when $r_{\text {tr }}$ changes from $66 r_{g}$ to $76 r_{g}$, which fit reasonably with the measured data.

We are grateful to an anonymous referee for helpful comments. This work was supported

by the Natural Science Foundation of China (under grant number 10873008) and the National Basic Research Program of China (973 Program 2009CB824800).

\section{REFERENCES}

Abramowicz, M. A., Bulik, T., Bursa, M., \& Kluźniak, W. 2003; A\&A, 404, L21

Abramowicz, M. A., Chen, X. M., Kato, S., Lasota, J. P., \& Regev, O. 1995, ApJ, 438, 37

Abramowicz, M. A. \& Kluźniak, W. 2001, A\&A, 374, L19

Aly, J. J. 1985, A\&A, 143, 19

Barret D., Kluźniak W., Olive J. F., Paltani S., \& Skinner G. K. 2005, MNRAS, 357, 1288 
Begelman, M. C. \& Pringle, J. E. 2007, MNRAS, 375, 1070

Brandenburg, A., Nordlund, A., Stein, Robert F., \& Torkesson, U. 1995, ApJ, 446, 741

Boutelier, M., Barret, D., \& Miller, M. C. 2009, MNRAS, 339, 1901

Cackett, E. M., Miller, J. M., \& Ballantyne, D. R. et al. 2009, ApJ submitted (arXiv:0908.1098)

Camenzind, M. 2007, Compact Objects in Astrophysics: White Dwarfs, Neutron Stars and Black Holes, Astronomy and Astrophysics Library, Berlin.

Cui, W., Zhang, S. N., \& Chen, W. 1998, ApJ, 492, 53

De Villiers, J. P. \& Hawley, J. F. 2003, ApJ, 589, 458

Esin, A., McClintock, J. E., \& Narayan, R. 1997, ApJ, 489, 865

Fu, W. \& Lai, D. 2009, ApJ, 690, 1386

Greiner, J., Cuby, J. G., \& McCaughrean, M. J. 2001, Nature, 414, 522

Hawley, J. F. 2000, ApJ, 528, 462

Hirose, S., Krolik, J. H., De Villiers, J. P., \& Hawley, J. F. 2004, ApJ, 606, 1083

Homan, J., Miller, J. M., Wijnands, R. et al. 2005, ApJ, 623, 383

Honma, F. 1996, PASJ, 48, 77

King, A. R., Pringle, J. E., \& Livio, M. 2007, MNRAS, 376, 1740

Kluźniak, W. \& Abramowicz, M. A. 2001, Acta Physica Polonica B, 32, 3605

Klźniak, W., Abramowicz, M. A., Kato, S., Lee, W. H., \& Stergioulas, N. 2004, ApJ, 603, L89

Kluźniak, Abramowicz, M. A., \& Lee, W. H. 2004, in X-ray Timing 2003: Rossie and Beyond, eds. Kaaret, Ph., Swank, J. H. \& Lamb, F. K. (AIP Conference Proceedings), 714, p. 379

Koide, S., Shibata, K., Kudoh, T., \& Meier, D. L. 2002, Science, 295, 1688

Koide, S. 2003, Physical Review D, 67, 104010

Komissarov, S. S. 2006, MNRAS, 368, 993

Lai, D. \& Tsang, D. 2009, MNRAS, 393, 979

Li, X. D. \& Zhang, C. M. 2005, ApJ, 635, L57

Liu, B. F., Yuan, W., Meyer, F., Meyer-Hofmeister, E., \& Xie, G. Z. 1999, ApJ, 527, 17

Manmoto, T. \& Kato, S. 2000, ApJ, 538, 295

Matsumoto, R. \& Tajima, T. 1995, ApJ, 445, 767 
McClintock, J. E. \& Remillard, R. A. 2006, in Compact Stellar X-ray Sources, eds. Lewin, W., van der Klis, M., Cambridge Astrophysics Series, No. 39, Cambridge, UK: Cambridge University Press, p.157

McClintock, J. E., Shafee, R., \& Narayan, R. et al. 2006, ApJ, 652,518

Miller, J. M., Wijnands, R., \& Homan, J. et al. 2001, ApJ, 563, 928

Morgan, E. H., Remillard, R. A., \& Greiner, J. 1997, ApJ, 482, 993

Moss, D. \& Shukurov, A. 2004 A\&A, 413, 403, C414.

Narayan, R. \& McClintock, 2008, NewAR, 51, 733

Perez, C. A., Silbergleit, A. S., Wagoner, R. V., \& Lehr, D. E. 1997, ApJ, 476, 589

Remillard, R. A. 2004, AIPC, 714, 13

Remillard, R. A. \& McClintock, J. E. 2006, ARA\&A, 44, 49

Remillard, R. A., McClintock, J. E., \& Sobczak, J. G. et al. 1999, ApJ, 517, L127

Rezzolla, L., Yoshida, S., Maccarone, T. J., \& Zanotti, O. 2003, MNRAS, 344, L37

Robinson, E. L., Wood, J. H., \& Wade, R. A. 1999, ApJ, 514, 952

RóżaŃska, A. \& Czerny, B., 2000, A\&A, 360, 1170

Ruediger, G., Elstner, D., \& Stepinski, T. F. 1995 A\&A, 298, 934.

Ruzmaikin, A. A., Turchaninov, V. I., Zeldovich, L. B., \& Sokoloff, D. D. 1979, Ap\&SS, 66, 369

Shafee, R., McClintock, J. E., Narayan, R. et al. 2006, ApJ, 636, 113

Shafter, A. W. \& Misselt K. A. 2006, ApJ, 644, 1104

Shi, C. S. \& Li, X. D. 2009, MNRAS, 392, 264

Stella, L., Vietri, M., \& Morskink, S. M. 1999, ApJ, 524, L63

Stone, J. M, Hawley, J. F., Gammie, C. F., \& Balbus, S. A., 1996, ApJ, 463, 656

Strohmayer, T. E. 2001a, ApJ, 552, L49

Strohmayer, T. E. 2001b, ApJ, 554, 169

Tassev, S. V. \& Bertschinger, E. 2008, ApJ, 686, 423

Torkelsson, U. 1998, MNRAS, 298, 55

Tout, C. A. \& Pringle, J. E., 1992 MNRAS, 259, 604.

Vishniac, E. T., Jin, L., \& Diamond, P. 1990, ApJ, 365, 648

Wagoner, R. V., Silbergleit, A. S., \& Ortega-Rodrguez, M. 2001, ApJ, 559, L25 
Wang, D., Ma, R., Lei, W., \& Yao, G. 2003, MNRAS, 344, 473

Wang, D., Ye, Y., Yao, G., \& Ma, R. 2005, MNRAS, 359, 36

Yuan, F. 2001, MNRAS, 324, 119 
Table 1: This table presents the masses ( $m$ in solar units), dimensionless spins $a$ for several BHs, and the inferred radii $\left(r_{\mathrm{tr}, \mathrm{u}}\right.$ and $\left.r_{\mathrm{tr}, 1}\right)$, the predicted frequency ratios $\left(\nu_{\mathrm{u}} / \nu_{1}\right)$ in the corresponding sources based on the measured upper and lower QPO frequencies $\left(\nu_{\mathrm{u}}\right.$ and $\nu_{1}$ in $\mathrm{Hz}$ ) respectively.

\begin{tabular}{lcccccccc}
\hline \hline Sources & $m$ & $a$ & $\nu_{\mathrm{u}}$ & $r_{\mathrm{tr}, \mathrm{u}}$ & $\nu_{\mathrm{u}} / \nu_{1}$ & $\nu_{1}$ & $r_{\mathrm{tr}, 1}$ & $\nu_{\mathrm{u}} / \nu_{1}$ \\
\hline GRO J1655-40 & 6.3 & 0.7 & 450 & 66.7528 & 1.5504 & 300 & 65.3002 & 1.5501 \\
GRS 1915+105 & 14 & 0.99 & 168 & 75.6114 & 1.5514 & 113 & 73.4984 & 1.5510 \\
GRS 1915+105 & 14 & 0.99 & 67 & 139.7920 & 1.5570 & 41 & 144.368 & 1.5572 \\
XTE J1550-564 & 9.6 & 1 & 276 & 69.8141 & 1.5503 & 184 & 68.2975 & 1.5500 \\
XTE J1550-564 & 9.6 & 0.5 & 276 & 69.8639 & 1.5515 & 184 & 68.3125 & 1.5512 \\
XTE J1550-564 & 9.6 & 0 & 276 & 69.9159 & 1.5526 & 184 & 68.3302 & 1.5524 \\
\hline
\end{tabular}




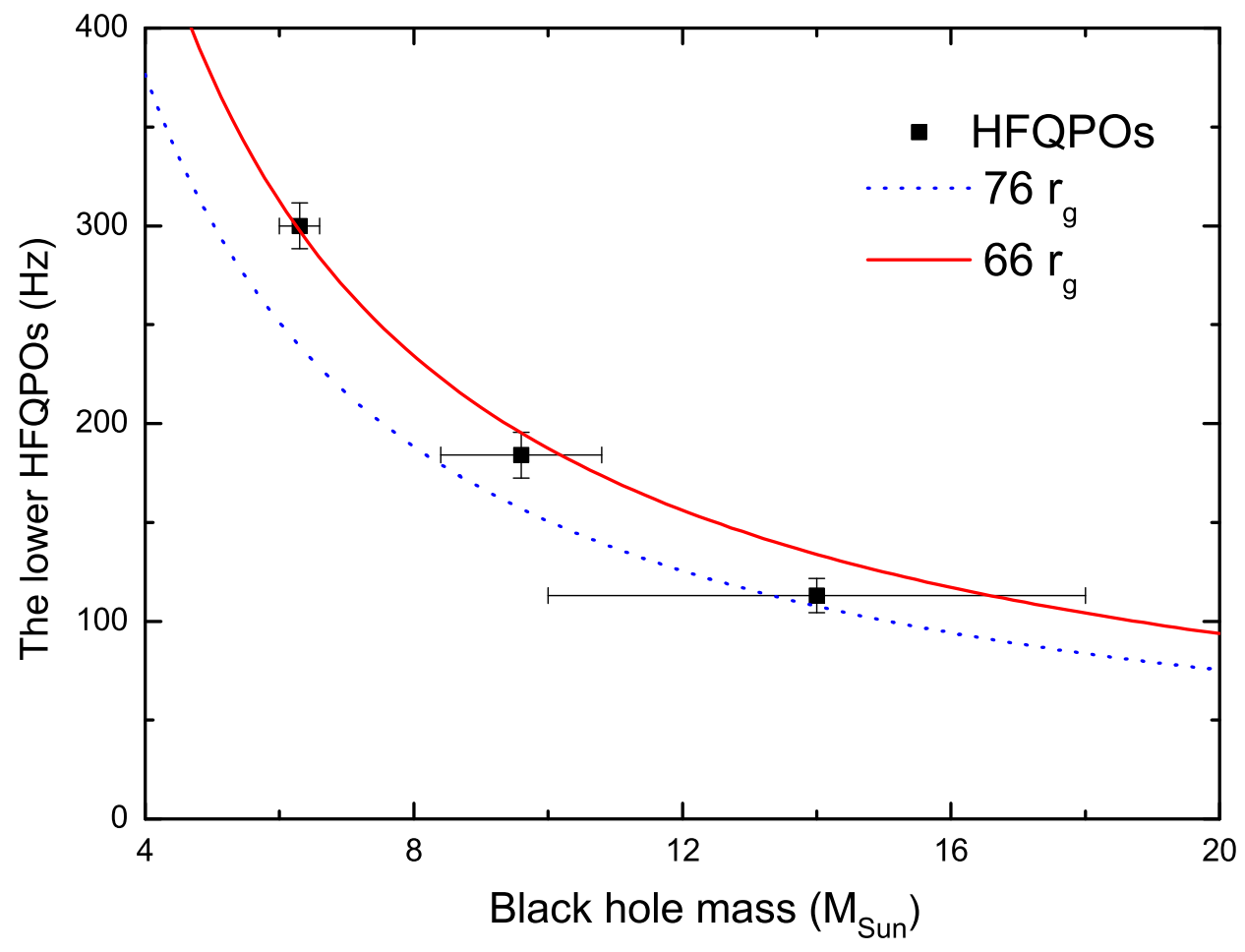

Fig. 1. - The relation between the lower HFQPO frequencies and BH masses for GRO J1655-40, XTE J1550-564, and GRS 1915+105. 